Proceedings of the 4th International Conference on the Structure of Surfaces

\title{
THE STRUCTURE OF SURFACES IV
}

Shanghai, China

August $16-19,1993$

Editors

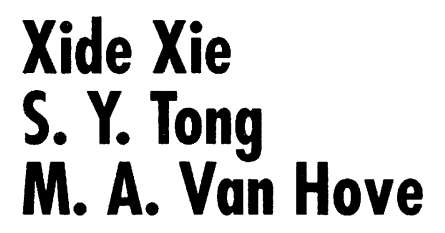

Shanghai, Milwaukee, Berkeley 


\section{CONTENTS}

Introduction $\quad \mathrm{v}$

Preface vii

Acknowledgement viii

Part I Techniques 1

Photoelectron Diffraction and Holography: Some New Directions 3

C. S. Fadley

Imaging of Surface Structure with Energy-Dependent Photoelectron

Diffraction

J. G. Tobin, G. D. Waddill, H. Li and S. Y. Tong

Theory of Scanning Tunneling Microscope as a Probe for Local

Electronic States of Surfaces

M. Tsukada, S. Watanabe, T. Schimizu and K. Hirose

Quantum Chemical Description of Tunnelling Experiments

P. Badziag and W. S. Verwoerd

New Vision on Surface Diffusion

X.-D. Xiao, Y. L. Xie and Y. R. Shen

An $A b$ Initio Photon-Stimulated-Desorption Theory

N. L. Liu and B. H. Choi

Surface Structure Effects on the Angular Anisotropy of Secondary Electrons Yield

S. Valeri, A. Di Bona, M. G. Lancellotti and E. Nava

Phase Transitions of Surfaces and Adsorbates Studied with Local Probes

R. Fink, G. Krausch and G. Schatz 
Coverage Dependent Adsorption Structures as Retrieved by

Conventional, Direct and Holographic DLEED Analysis

K. Heinz

Anisotropic Temperature Factors in the Calculation of Low-Energy Electron Diffraction Intensities

W. Moritz and H. Over

A New Approach to the Automatic Solid Structure Determination

S. Nagano

Relaxations and Reconstructions Derived from SEXAFS Spectra of Adsorbates on Single Crystal Surfaces

N. Binsted and D. Norman

Theoretical Analysis of Carbon K-Edge NEXAFS Spectra of Graphitelike Film and Related Material

Y. Zou, X. S. Feng and J.C. Tang

A Fast Simulation Program for a Full Analysis of LEIS Data

G. Dorenbos and D. O. Boerma

The Effects of Loss on Surface Electron Spectroscopy: Theoretical Study

T. Fujikawa

\section{Part II Clean Metals}

Mechanisms and Energetics of Surface Atomic Processes

T. T. Tsong

Thermal Phase Behavior of Metal Surfaces: X-Ray Scattering Studies of $\mathrm{Pt}(001)$

D. L. Abernathy, D. Gibbs, G. Grübel, S. G. J. Mochrie,

G. M. Watson, M. Yoon and D. M. Zehner

Surface Energy Anisotropy and Equilibrium Crystal Shape of Metals

S. Q. Wei and M. Y. Chou

STM Study of Vicinal Structures on Au(100)

Y. Samson, S. Rousset, S. Gauthier, J. C. Girard and J. Klein 
Thermal Studies of Bulk Copper Surface by Extended Fine Auger Structure Spectroscopy

L. Lozzi, M. Passacantando, P. Picozzi, S. Santucci and M. De Crescenzi

Stress-Induced Microfacetted Reconstructions of the $\mathrm{Pb}(110)$

Surface

M. S. Altman, S. Chiang, P. Statiris, T. Gustafsson and E. Bauer

\section{Part III Metals with Adsorbates}

Initial Stage of Diamond Film Formation on Platinum(111) Surface

K. A. Feng and Z. D. Lin

Dynamical LEED Studies of Ethylidyne on $\mathrm{Pt}(111)$ and $\mathrm{Rh}(111)$ :

Comparison of Molecular Distortions and Substrate Relaxations

A. Barbieri, M. A. Van Hove and G. A. Somorjai

Ambiguities in Adsorbate Site Assignment from Vibrational Frequencies Revealed by LEED

L. D. Mapledoram, A. Wander, P. Hu and D. A. King

The Structures of $\mathrm{H}_{2} \mathrm{O}$ and $\mathrm{D}_{2} \mathrm{O}$ Adsorbed on $\mathrm{Ru}(001)$

G. Held and D. Menzel

CO Adsorption on $\mathrm{Ni}_{3} \mathrm{Ti}(001)$ and $\mathrm{Ti}$-Oxidized $\mathrm{Ni}_{3} \mathrm{Ti}(001)$

L. J. Yao, G. X. Xiong, D. Z. Wang, X. M. Wei and X. X. Guo

The Structural and Dynamical Properties of Molecularly Adsorbed $\mathrm{O}_{2}$ on $\mathrm{Cu}(100)$

T. Yokoyama, D. Arvanitis, T. Lederer, G. Comelli, M. Tischer,

L. Tröger and K. Baberschke

Multiple Scattering Studies of the C-H Resonances in X-Ray

Absorption Fine Structure of HCOO-Cu(110)

J. C. Tang, X. S. Feng and Y. Zou

The Local Adsorption Structure of Acetylene on $\mathrm{Cu}(111)$

S. Bao, K.-M. Schindler, Ph. Hofmann, A. M. Bradshaw and

D. P. Woodruff 
Multiple-Scattering Approach to K-Edge NEXAFS of Cyclopentadienyl on $\operatorname{Pt}(111)$ Surface

M. Yimagawa and T. Fujikawa

A Structure Transition of Ethanol on Silver Surface Studied by Laser Spectroscopy Photoacoustic

A. R. Zhu, C. W. Hu and Z. Y. Wang

The Geometry of $\mathrm{CH}_{3} \mathrm{NO}_{2}$ Adsorbed on the $\mathrm{Cu}(111)$ Surface

$X$. S. Zhang and Z. Du

Adsorption and Decomposition of Methanol on Clean and Oxygen Preadsorbed Pd(100)

C. L. Su, Z. J. $Q u, X$. J. Ding, S. K. Shen, X. M. Wei, K. Wu,

Y. M. Cao and D. Z. Wang

Multiple-Scattering Analysis of Propargyl Alcohol and Propargyloxy Adsorbed on $\mathrm{Ag}(110)$ Surface

X. S. Feng, Y. Zou and J. C. Tang

The Effect of Ti Intermediate Layer Made by IBAD on the Cu Surface Film of E-gun Evaporation J. Yang, C. Wang, K. Tao and Y. D. Fan

$\mathrm{H}_{2}$ Dissociative Adsorption on the Imperfect $\mathrm{Ni}(100)$ Surface J. J. Xie, P. Jiang and K. M. Zhang

Charge-Exchange Scattering of Alkali Ions/Atoms from Metal Surfaces

L. M. Pan, Y. S. Wang, F. Y. Huang, D. F. Fang, J. Y. Tang and F. J. Yang

Time-Dependent Quantum Dynamics Treatment of Dissociation Chemisorption of $\mathrm{H}_{2}$ on W(110) Surface 301 $X$. Zhao and D. Guan

Compound Formation and Ultrathin Amorphous Layers at In/Pd-Interfaces Observed by Local and Non-Local Techniques R. Fink, T. Koch, G. Krausch, J. Marien, A. Plewnia, B.-U. Runge, G. Schatz, A. Siber and P. Ziemann 
Relationship between Molar Heat of Adsorption and Microscopic Properties of the Adsorbed Molecule

C. H. Yang

Scaling in the Growth of $\mathrm{Pb}$ Islands on $\mathrm{Cu}(001)$

G. Vidali, O. Biham, H. Zeng, J.-S. Lin and W. Li

The Structure of the Domain-Wall Lattice in $\mathrm{Pb} / \mathrm{Cu}(110)$ System:

Surface X-Ray Diffraction Studies

C.-H. Lee and K. S. Liang

Microscopic Aspects of Faceting Induced by Ultrathin Metal Films:

a Comparison of $\mathrm{Pd} / \mathrm{W}(111)$ and $\mathrm{Pd} / \mathrm{Mo}(111)$

C.-Z. Dong, J. Guan, R. A. Campbell and T. E. Madey

Observation of a New Class of Reconstructions: An Adsorbate Stabilised Vacancy Structure for $\mathrm{c}(2 \times 2) \mathrm{X}-\mathrm{W}(100)$

A. Wander, P. Hu, M. P. Bessent and D. A. King

\section{Part IV Chemisorbed Semiconductors}

Scanning Tunneling Microscopy (STM) of Si/Ge-Layers on Si(100) and Subsequent Photoluminescence (PL) of Si/Ge Quantum Wells Grown by Si-Molecular Beam Epitaxy

R. Butz, A. Hartmann and H. Lüth

Azimuthal Variation of the Step Distribution on Vicinal Si(001)

Surfaces

J. Waßerfall and W. Ranke

Theoretical Analyses of Thermal Desorption Spectra of Alkali Adsorbed Si(100) Surface

K. Kakitani, J. -L. Wang and A. Yoshimori

Fine Structure in the $\Delta \phi(\theta)$ Plot of the $\mathrm{Na} / \mathrm{Si}(111)-7 \times 7$ System

R. S. Li, K. Kojima, T. Yasue and T. Koshikawa

RHEED-TRAXS Study of Surface Structure and Thermal Desorption of $\mathrm{Cu}$ on $\mathrm{Si}(111)$ Surface

Z. H. Zhang, S. Hasegawa and S. Ino 
xiv

Correlation between Atomic-Scale Structures and Macroscopic Electrical Properties of Metal-Covered Si(111) Surfaces Investigated by in-situ Measurements in UHV

S. Hasegawa and S. Ino

In-Plane Structure of Si(111): As-1 $\times 1$ Surface Studied Using Grazing-Angle X-Ray Standing Waves

O. Sakata and H. Hashizume

Annealing Study of $\mathrm{Au} / \mathrm{GaAs}(110)$ Interface Using Low Energy Positron Beam

H. M. Weng, J. H. Xu, S. J. Sun, R. D. Han, C. C. Ling, Y. Y. Shan,

T. C. Lee, S. Fung and C. D. Beling

Restructuring of the $\mathrm{Si}(111) 7 \times 7$ Surface upon Adsorption of $\mathrm{Ag}$

A. Shibata and K. Takayanagi

Study of Atomic Depth Distribution and Growth Modes During Epitaxial Growth of $\mathrm{Sn}$ on $\mathrm{Si}(111)-\sqrt{3} \times \sqrt{3}-\mathrm{Ag}$ by RHEED-TRAXS (Total Reflection Angle X-Ray Spectroscopy)

S. Ino and T. Yamanaka

Structure Analysis of $\mathrm{Si}(111)-\sqrt{3} \times \sqrt{3}-\mathrm{Ag}$ by RHEED 408

A. Ichimiya, M. Ando, S. Kohmoto and Y. Horio

A Comparative Study of the Structures of $\mathrm{Ag} / \mathrm{Si}(111)-\sqrt{3} \times \sqrt{3}$ and $\mathrm{Ag} / \mathrm{Ge} / \mathrm{Si}(111)-\sqrt{3} \times \sqrt{3}-\mathrm{Ag}$ Surfaces

T. Abukawa, N. Nakamura, T. Goto and S. Kono

STM and Synchrotron Radiation Studies of the Surface Structures of $\mathrm{Ag} / \mathrm{Ge}(111)$

M. Gothelid, M. Hammar, U. O. Karlsson, S. A. Flodstrom,

L. Seehofer, T. Buslaps, R. L. Johnson, E. Findeisen,

R. Feidenhans'l, M. Nielsen, V. Yu. Aristov and G. Le Lay

LEED Analysis of $\mathrm{Ge}(111)(\sqrt{3} \times \sqrt{3}) \mathrm{R} 30^{\circ}-\mathrm{Ag}$ and $\mathrm{I}(\mathrm{V})$ Comparisons

H. Zhao, D. M. Hobday and S. P. Tear

Influence of Surface Termination on Visible-Light Emission of Porous Silicon: A Comprehensive Luminescence Model

J. Wang, W.-C. Wang, J.-B. Zheng, Z.-M. Zhang, F.-L. Zhang, $X$. - Y. Hou and $X$. Wang 
An Explanation of the Temperature Variation of Atomic Structure on $\mathrm{Si}(113)$ Surface

J.-A. Wu, J.-P. Zhang and Y.-R. Xing

Adsorption Structures and Dissociation Properties of $\mathrm{PH}_{3}$ on $\operatorname{Si}(100)-2 \times 1$ and $\operatorname{Si}(111) 7 \times 7$

P. L. Cao, J. J. Dai and R. H. Zhou

The Effect of Oxidation on Surface Structure of Silicon Observed with Scanning Tunneling Microscopy

Q. Cai, C. W. Hu, S. Z. Dong, Y. F. Zhang and A. R. Zhu

LEED I(V) Analysis of the Si(111)-Pb System and Study of the $\mathrm{Si} / \mathrm{Pb} / \mathrm{Si}(111)$ System

H. Zhao, A. H. Jones and S. P. Tear

Theoretical Study of a Iron Monolayer on $\beta$-SiC(100) Surfaces

W. C. Lu, K. M. Zhang and X. D. Xie

InP(100) Surface Structural Changes Induced by Ion Sputtering $\left(\mathrm{Ar}^{+}\right)$ and Adsorbate(Sb) Deposition Probed by Photoemission EXAFS 466

P. S. Mangat, Y. Huttel, B. Gruzza, A. Porte, Z. Hurych and P. Soukiassian

A Study of Adsorption of Rb and K Atoms on InP(100) Surface with DV-X $X_{\alpha}$ Method

P. S. Xu, S. H. Xu, J. S. Zhu, X. M. Liu, M. S. Ma, Y. H. Zhang and $Z . J . X u$

The Study of Low Temperature Growth InSb on Tilted GaAs Substrates

L. Zhang, H. Chen, N. Luo, Q. Huang, J. M. Zhou and F. H. Li

Structure and Composition of Sulfur Passivated GaAs Surface X. Wong, X.-Y. Hou, Z. S. Li, W. Z. Cai and G. S. Dong

Assessing Surface Reconstructions by Angle-Scanned X-Ray

Photoelectron Diffraction

M. Seelmann-Eggebert, R. Klauser, G. P. Carey, E. C. Larkins, P. Meisen and H. J. Richter 
xvi

Growth Steps in the Interface of Short Period AlAs/GaAs

Superlattices

H. Chen, Y. Huang, J. M. Zhou and F. H. Li

Effect of Surface Condition on the Electronic and Optical

Properties of Silicon Nanostructures

504

H. X. Fu, L. Ye and X. D. Xie

\section{Part V Oxides and Alloys}

Experimental Study on the Melting Transition at Interface between Ice Crystal and Substrate

Y. Furukawa, T. Ishizaki, I. Ishikawa and M. Maruyama

Commensurate Structures of $\mathrm{CO}$ on $\mathrm{MgO}(100)$ Explored by High Resolution LEED

J. Suzanne, V. Panella and M. Sidoumou

Calculation of Commensurate Monolayer Structures: CO Adsorbed on $\mathrm{NaCl}(100)$ and $\mathrm{MgO}(100)$

C. Girardet and P. N. M. Hoang

A Special Case of an Overlayer Structure: the Ni-0.8\% $\mathrm{Au}(110)$ Alloy Surface

G. Dorenbos, D. O. Boerma, T. M. Buck and G. H. Wheatley

The Atomic Structure of Cobalt Deposited on the Pt(111) Surface

A. Atrei, U. Bardi, M. Galeotti, G. Rovida, M. Torrini and

E. Zanazzi

Molecular Dynamics Study of Surface Melting on Ice Crystal

Effect of Surface Structure on Diffusion of Ag in High Temperature Superconductor $\mathrm{YBa}_{2} \mathrm{Cu}_{3} \mathrm{O}_{7-\delta}$

P. P. Gorbik, I. V. Dubrovin, V. V. Dyakin, G. N. Kashin and

V. I. Makhnjuk

Coadsorption of Oxygen and Carbon Monoxide on Ordered Alloy Surface Pd $\{001\} \mathrm{c}(2 \times 2)$-Mn Studied by HREELS

Jia, K. Wu, S.-H. Lu, R.-G.-F. Zhao, D. Z. Wang and S.-C. Wu 
Study of the Adsorption and the Diffusion of Hydrogen on the Surface of TiAl Alloy by AP-FIM

D. G. Ren and M. Z. Cao

The HREELS and ARUPS Investigation of CO Adsorption on

$\mathrm{Fe} / \mathrm{Cu}(110)$ Bimetallic Surface

C. Y. Fan, S. N. Bao, H. Y. Li and Y. B. Xu

Niobium Oxide Crystallites Epitaxially Grown on Pt(111)

L. Xie, D. Z. Wang, C. M. Zhong, X. X. Guo, T. Ushikubo and

K. Wada

Multiphase Surface Morphologies of Homoepitaxial LPCVD

Diamond Films Using Scanning Probe Microscopies

H. G. Maguire

Outermost Atomic Layer on Antifluoritic $\mathrm{Li}_{2} \mathrm{O}(111)$

593

W. P. Ellis, R. Bastasz and I. Shindo

Theoretical and Experimental Study of the Surface Structure of $\mathrm{MgO}(001)$

J. B. Zhou, Y. Li, H. C. Lu, D. Langreth, T. Gustafsson,

$P$. Häberle and M. R. Pederson

\section{Part VI Growth and Epitaxy}

Local Structural Study of Ultrathin Metal Films on $\mathrm{TiO}_{2}(110)$

Using ARXPS and MEED

J.-M. Pan, B. L. Maschhoff, U. Diebold and T. E. Madey

Modes in Homoepitaxial Growth on $\mathrm{Cu}(111), \mathrm{Ag}(111)$ and $\mathrm{Pt}(111)$

M. Henzler, T. Schmidt and E. Z. LuO

Growth of Thin Au Films on Ni(001)

P. Statiris and T. Gustafsson 


\title{
Anisotropic temperature factors in the calculation of low-energy electron diffraction intensities
}

\author{
W. Moritz and $\mathrm{H}$. Over*
}

Institut für Kristallographie und Mineralogie, Universität München, Theresienstr. 41, 80333 München, Federal Republic of Germany

*Fritz-Haber-Institut der Max-Planck-Gesellschaft, Faradayweg 4-6, 14195 Berlin, Federal Republic of Germany

\begin{abstract}
A theory is presented to introduce anisotropic and anharmonic vibrations into the multiple scattering formalism based on a spherical wave expansion of the probabilty density of the atoms. This results in atomic $t$ - matrices with off-diagonal elements instead of the usual diagonal matrices valid for spherical symmetric vibrations. The atomic $t$ - matrices can be inserted in the conventional multiple scattering formalism. The importance of anisotropic vibrations in a LEED $\mathrm{L} / \mathrm{V}$ analysis is demonstrated with a simplified calculation where anisotropic vibrations are approximated by split positions. This concept has been applied to $\mathrm{CO} / \mathrm{Ru}(001)-(\sqrt{3} \mathrm{x} \sqrt{3})$ where a considereable improvement of the structure refinement could be obtained with an anisotropic vibration amplitude attributed to a bending mode of the $\mathrm{CO}$ molecule.
\end{abstract}

\section{Introduction}

Thermal vibrations are of fundamental interest in understanding the physical and chemical properties of surfaces. Vibration amplitudes are expected to be considerably larger on surfaces than in the bulk and it can be safely assumed that anharmonic terms in the thermal vibrations are also larger than in the bulk. It would be highly desirable to have a reliable method which allows the quantitative determination of the anisotropy of thermal vibration amplitudes. Surface diffusion plays an important role in surface reactions and the measurement of the anharmonicity would be largely of interest as well. Unfortunately there exist only few methods to determine quantitatively the vibration amplitudes and their spatial anisotropy. LEED, like X-ray diffraction, would be well capable to study thermal 
vibrations but with very few exceptions only isotropic vibrations have been considered in the multiple scattering theory until now.

In the multiple scattering theory thermal vibrations are usually treated in the Debye approximation. This approximation has been introduced into the commonly used formulation of the multiple scattering theory in a spherical wave representation. It requires spherical symmetric thermal vibrations and the Einstein model of independent vibrations. This treatment of thermal vibrations usually works well in the case that only the static structure is of interest. as it has often been found that there is little influence of the thermal parameters on the structural result. Therefore the optimisation of thermal vibrations is often not performed and the vibration amplitudes derived from the Debye model are, in turn, usually not very reliable. It is also well recognised that the Debye model and the muffin tin approximation for the crystal potential is limiting the accuracy of the LEED $\mathrm{I} / \mathrm{V}$ analysis.

It arises the question whether the quantitative determination of vibration amplitudes with LEED is at all possible or not. The shortcomings of the multiple scattering theory may outweigh the influence of the approximations made with the thermal vibrations. The other main approximations made in the conventional multiple scattering calculations are the muffin tin approximation for the crystal potential and the isotropic damping model for the inelastic scattering. Only a few attempts have been tried to include non muffin tin corrections in the multiple scattering theory $[1,2]$. It can be expected that details of the potential are important at low energies. The low energy range, on the other hand, is certainly not appropriate to study thermal effects because of the low sensitivity at small momentum transfer. In the higher energy range, lets say above $100-150 \mathrm{eV}$, the errors in the crystal potential can be considered of minor influence and thermal vibrations will be dominant. We can therefore expect that the effects of anisotropic potentials and anisotropic vibrations, which are certainly strongly correlated, could be separated in a LEED I/V analysis at least when temperature dependent measurements are included.

To gain insight into the importance of thermal vibrations in the LEED analysis it is useful to compare LEED with X-ray diffraction where the kinematical approximation can be applied successfully. Here the use of isotropic vibrations usually leads to a fairly good structural result but a poor $\mathrm{R}$-factor. The position parameters change in most cases only slightly after including anisotropic vibrations but the R-factor, or the GOF (goodness of fit), drops frequently considerably. It is also not uncommon that different structural 
models can only be distinguished after considering anisotropic vibrations. Though the quantitative determination of vibration amplitudes by $\mathrm{X}$-ray diffraction is often of severely hindered by absorption effects it is usually not possible to neglect anisotropic vibrations. We certainly cannot expect that anisotropic vibrations play a minor role in the case of LEED than in the case of X-ray diffraction.

To investigate how much anisotropic vibrations would influence the LEED I/V curves we have applied the concept of split positions to the system $\mathrm{CO} / \mathrm{Ru}(001)$ [3]. This system has been subject of a previous LEED structure analysis [4]. We chose this system because of the quality of the data and because large anisotropic effects can be expected here. Furthermore, the bending mode of the $\mathrm{CO}$ molecule has been studied by ESDIAD measurements [5-7] and we can compare the LEED results with independent measurements. The results are promising and show that the inclusion of anisotropic vibrations should lead to a significant improvement of the LEED I/V analysis.

\section{Theory}

The diffracted intensity can be written using the T-matrix formalism as

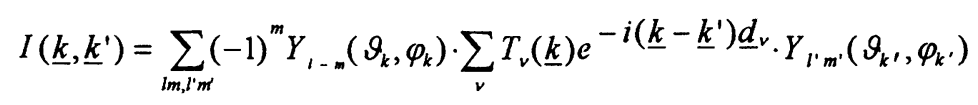

where the T-matrices are given by the solution of a self-consistent equation

$$
T_{\nu}(\underline{k})=t_{\nu}(E)+t_{\nu}(E) \cdot \sum_{\mu} G_{\nu \mu}\left(\underline{k}, \underline{P}+\underline{d}_{\mu}-\underline{d}_{\nu}\right) T_{\mu}(\underline{k})
$$

The definition of the matrices $\mathrm{T}$ for the multiple scattering process, $t$ for the single scattering process and the electron propagator functions $\mathrm{G}(\underline{\underline{k}}, \underline{\mathrm{P}})$ can be found in the book by Pendry [8]. $\underline{k}$ and $\underline{k}^{\prime}$ are the wave vectors of the incoming and outgoing wave and $\mathrm{Y}_{\mathrm{Im}}$ are the spherical harmonics. Introducing thermal vibrations the diffracted intensity is given by

$$
I\left(\underline{k}, \underline{k}^{\prime}\right)=\sum_{l m^{\prime} r m^{\prime}}(-1)^{m} Y_{1, m}\left(\vartheta_{k}, \varphi_{k}\right) \cdot\left\langle\sum_{\nu} T_{\nu}\left(\underline{k}, \underline{d}_{\nu}+\Delta \underline{d}_{\nu}\right) e^{-i\left(\underline{k}-\underline{k}^{\prime}\right)\left(\underline{d}_{\nu}+\Delta \underline{d}_{\nu}\right)}\right\rangle_{T} \cdot Y_{l^{\prime} m^{\prime}}\left(\vartheta_{k^{\prime}}, \varphi_{k^{\prime}}\right)
$$

where the average indicated by \langle\rangle$_{\mathrm{T}}$ is taken over the thermal distribution of displacements $\Delta \mathrm{d}_{\mathrm{v}}$. The individual $\mathrm{T}$-matrices at each moment with displacements $\Delta \mathrm{r}_{v}$ are given by 


$$
T_{\nu}\left(\underline{k}, \underline{d}_{\nu}\right)=t_{\nu}(E)+t_{\nu}(E) \cdot \sum_{\mu} G_{\nu \mu}\left(\underline{k}, \underline{d}_{\mu}-\underline{d}_{\nu}-\Delta \underline{d}_{\nu}+\Delta \underline{d}_{\mu}\right) \cdot T_{\mu}\left(\underline{k}, \Delta \underline{d}_{\mu}\right)
$$

To solve this equation the thermal average has to be introduced. We define a temperature dependent $\mathrm{T}$ matrix which is a function of the distribution of the displacements

$$
T_{v}(\underline{k}, T)=\left\langle T_{v}\left(\underline{k}_{,} \underline{d}_{v}+\Delta \underline{d}_{v}\right) e^{\left.-i\left(\underline{k}-\underline{k}^{\prime}\right) \Delta \underline{d}_{v}\right\rangle_{T}}\right.
$$

and likewise for the single scattering amplitude

$$
t_{v}(E, T)=\left\langle t(E) \cdot e^{i(\Delta \underline{k} \cdot \Delta \underline{d})}\right\rangle_{T}
$$

Correlations between atomic displacements as well as correlations in the multiple scattering series are neglected here. It has been shown first by Duke and Laramore [9], Holland [10] and by Pendry [8] that in the harmonic approximation and assuming isotropic vibrations equ. 4 can be simplified to

$$
T_{\nu}(\underline{k}, T)=t_{\nu}(E, T)+t_{\nu}(E, T) \cdot \sum_{\mu} G_{\nu \mu}\left(\underline{k}, \underline{d}_{\mu}-\underline{d}_{\nu}\right) \cdot T_{\mu}(\underline{k}, T)
$$

where $t_{v}(E, T)$ can be put in a diagonal form defining complex, temperature dependent phase shifts. To include anharmonic and anisotropic vibrations one has to go back to eqs. 4 and 5 and to introduce the appropriate averages. This is best done introducing a probabilty density for the atomic displacements. It means that the electron is scattered by a spherical symmetric potential which is displaced. It is not necessary nor convenient to describe anisotropic vibrations by non-muffin-tin corrections to the atomic potential which have been introduced into the multiple scattering theory by Nagano and Tong [1]. The average over atomic displacements is not restricted to harmonic vibrations and allows to include anharmonic terms as well. We define a probability $p(r)$ of finding an atom at position $r$ by

$$
p(r)=\sum_{n, m . l} R_{n}(r) \cdot c_{n l m} \cdot Y_{l m}\left(\vartheta_{r}, \varphi_{r}\right)
$$

where $c_{\text {nlm }}$ are appropriate anisotropy factors which can be determined from the experimental data in a fit procedure. For $R_{n}(r)$ usually a Gaussian distribution can be taken and only one value of $\mathrm{n}$ is required. However, in the general case a combination of 
several distribution functions becomes necessary. The number of free parameters is limited as in most cases only quadrupole or octupole terms need to be considered which limits $l$ to 2 or 3 . Further reduction of the number of free parameters is obtained by symmetry relations. This approach differs from an earlier attempt to include anisotropic vibrations in the multiple scattering formalism by Ulehla and Davis [11] where the anisotropy between parallel and normal components was treated in rectangular coordinates and led to large computational effort. The temperature dependent atomic scattering factor for single scattering is now given by

$$
t\left(T, \underline{k}, \underline{k}^{\prime}\right)=t\left(0, \underline{k}, \underline{k}^{\prime}\right) \cdot \int p(\Delta \underline{r}) \cdot e^{i\left(\underline{k}-\underline{k}^{\prime}\right) \Delta \underline{r}} d \Delta \underline{r}
$$

The atomic scattering factor at $\mathrm{T}=0$ can be written as a diagonal matrix

$$
t\left(0, k, k^{\prime}\right)=\frac{-8 \pi^{2}}{k} \sum_{l m, l^{\prime} m} e^{i \eta_{l}} \cdot \sin \left(\eta_{l}\right) \cdot \delta_{l l^{\prime}} \cdot \delta_{m m^{\prime}} \cdot Y_{l m}\left(\vartheta_{k}, \varphi_{k}\right) \cdot Y_{l^{\prime}-m^{\prime}}\left(\vartheta_{k^{\prime}}, \varphi_{k^{\prime}}\right) \cdot(-1)^{m^{\prime}}
$$

and for the phase factors a spherical wave expansion can be used.

$$
e^{i(\underline{k} \cdot \underline{r})}=\sum_{l m} 4 \pi \cdot i^{l} \cdot j_{l}(k r) \cdot Y_{l m}\left(\vartheta_{r}, \varphi_{r}\right) \cdot Y_{l-m}\left(\vartheta_{k}, \varphi_{k}\right) \cdot(-1)^{m}
$$

Utilising the properties of the spherical harmonics we obtain

$$
\int p(\Delta \underline{r}) \cdot e^{i \Delta \underline{k} \cdot \Delta \underline{r}} \cdot d r=\sum_{L, M} \sum_{L^{\prime}, M^{\prime}} W_{L M, L^{\prime} M^{\prime}} \cdot Y_{L M^{\prime}}\left(\vartheta_{k}, \varphi_{k}\right) \cdot Y_{L^{\prime}-M^{\prime}}\left(\vartheta_{k^{\prime}}, \varphi_{k^{\prime}}\right) \cdot(-1)^{M^{\prime}}
$$

with

$$
\begin{aligned}
W_{L M, L^{\prime} M^{\prime}}= & 16 \pi^{2} \cdot \sum_{n, l m} B_{L M, L^{\prime} M^{\prime}, l m} \cdot c_{n, l m} \\
& \cdot \int R_{n}(\Delta r)(i)^{L+L^{\prime}} \cdot j_{L}(k \cdot \Delta r) \cdot j_{L^{\prime}}\left(k^{\prime} \cdot \Delta r\right) \cdot(\Delta r)^{2} \cdot d \Delta r
\end{aligned}
$$

Defining temperature dependent expansion coefficients of the scattering factors and following the derivation given by Pendry [8]

$$
t\left(T, \underline{k}, \underline{k}^{\prime}\right)=8 \pi^{2} \sum_{L M} \sum_{L^{\prime} M^{\prime}} t_{L M, L^{\prime} M^{\prime}}(T) \cdot Y_{L, M}\left(\vartheta_{k}, \varphi_{k}\right) \cdot Y_{L^{\prime},-M^{\prime}}\left(\vartheta_{k^{\prime}}, \varphi_{k^{\prime}}\right) \cdot(-1)^{M^{\prime}}
$$


we obtain

$$
t_{L M, L^{\prime} M}(T)=W_{L M, L^{\prime} M} \cdot D_{L M, L^{\prime} M}
$$

where

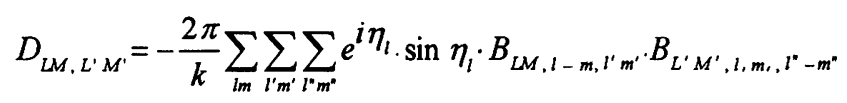

are temperature independent factors and

$$
B_{L M, l^{\prime} m^{\prime}, l m}=\int Y_{L M}(\Omega) \cdot Y_{l^{\prime}-m^{\prime}}(\Omega) \cdot Y_{l m}(\Omega) \cdot d \Omega
$$

For spherical symmetric vibrations all coefficients $c_{n, l m}$ vanish unless $1=0$ leading to a diagonal form of the matrices $t_{L M \mathcal{L}^{\prime} M^{\prime}}$ as it should be. In the case of anisotropic vibrations the matrices contain off-diagonal elements. Their calculation is not time consuming. The integral in equ. 13 can be solved numerically where the parameters $c_{n l m}$ and the parameters of the radial distribution function are subject of an optimisation procedure.

\section{'Split'-positions}

A complete description of the theory outlined above and further applications will be given in a forthcoming publication. Here we describe only an approximation where the average in equ. 5 is replaced by a sum over discrete displacements $\Delta r$. This serves as a check of the importance of anisotropic thermal vibrations in the LEED I/V analysis. Partially occupied positions are usually applied in X-ray structure analysis in cases where certain atoms occupy discrete positions in a statistical distribution and are conventionally called split positions. In the kinematic theory split positions cannot be distinguished from anisotropic vibrations as long as the displacements are much smaller than an atomic diameter and the momentum transfer is small. At high momentum transfer the damping of the reflection intensities by the temperature factor are not correctly described by split positions. It means that the average

$$
\left\langle e^{i \Delta \underline{k} \cdot \Delta \underline{r}_{T}}\right\rangle_{T} \approx e^{-\frac{1}{2}\left\langle(\Delta \underline{k} \cdot \Delta \underline{r})^{2}\right\rangle_{T}}
$$


is replaced by a discrete sum with a small number of positions $r_{\alpha}$

$$
\left\langle e^{i \Delta \underline{k} \cdot \Delta \underline{r}}\right\rangle_{T}=\sum_{\alpha} p_{\alpha} \cdot e^{i \Delta \underline{k} \cdot \Delta \underline{r}_{\alpha}}
$$

which is justified as long as the exponential is small. Split positions can be easily introduced into the conventional multiple scattering calculation. Care has to be taken to exclude multiple scattering paths between split positions which cannot be occupied simultaneously. Each position corresponds to an independent atom in a composite layer and the whole calculation becomes fairly lengthy such that is it not possible to introduce a realistic probability density for the displacements. The advantage is that full use can be made of the symmetry of the average structure.

An alternative approach is to neglect the average in the propagator matrices in equ. 4 and to solve equ. 2 instead for a displaced atom and to average the diffracted amplitudes. It has the advantage that a distribution density can be introduced and the amplitudes $\mathrm{A}\left(\mathrm{k}, \mathrm{k}^{\prime}\right)$ can be calculated by TLEED as proposed by Pendry and Heinz [12]. The disadvantage here is that the symmetry is broken and that the intra-layer multiple scattering is calculated for a rigid lattice. The diffracted intensity is then given by

$$
I\left(k, k^{\prime}\right)=\left|\int p(\Delta r) \cdot A\left(k, k^{\prime}, \Delta r\right) d \Delta r\right|^{2}
$$

The integral can be replaced by a sum over a sufficient number of discrete positions. The method will work well in cases where intra-layer multiple scattering is small. In the present case of $\mathrm{CO} / \mathrm{Ru}(001)-(\sqrt{3} \mathrm{x} \sqrt{3})$ it leads to nearly identical results with the split positions which is expected because of the wide spacing between the $\mathrm{CO}$ molecules and the normal incidence. In the kinematic limit both methods are identical.

\section{Application to $\mathrm{CO} / \mathrm{Ru}(001)-(\sqrt{3} \mathbf{x} \sqrt{3})$}

We apply the concept of split positions to study the thermal vibrations of the $\mathrm{CO}$ molecule on $\mathrm{Ru}(001)$. Before applying split positions to the $\mathrm{O}$ and $\mathrm{C}$ atoms the structure analysis has been redone to ensure that all static parameters were at optimum values because in the previous analysis [4] the relaxation in the Ru-layer could not be considered. The analysis 
included lateral and vertical relaxation in the 2 uppermost substrate layers and resulted in a small buckling in the top Ru layer.

\section{$\mathrm{CO} / \mathrm{Ru}(0001) \vee 3 \times \vee 3 \mathrm{R} 30^{\circ}$}
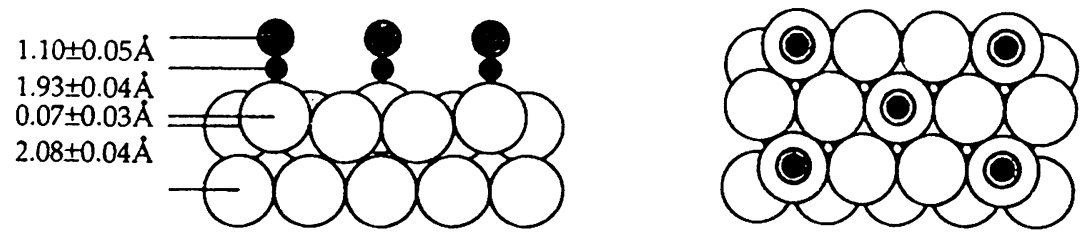

Fig. 1 Structure model of the $C O(R u(001)-(\sqrt{3} x \sqrt{3})$ phase

The $\mathrm{Ru}$ atoms co-ordinated to the $\mathrm{C}$ atom are displaced outward by about $0.07 \AA$. No lateral displacements could be detected within the error limits. The $\mathrm{R}$-factors decreased significantly, $R_{P}$ dropped from 0.51 to 0.34 and $R_{D E}$, which corresponds to $R_{1}$ dropped from 0.44 to 0.32 . Fig. 1 shows the structure model and the resulting parameters.

split positions of oxygen
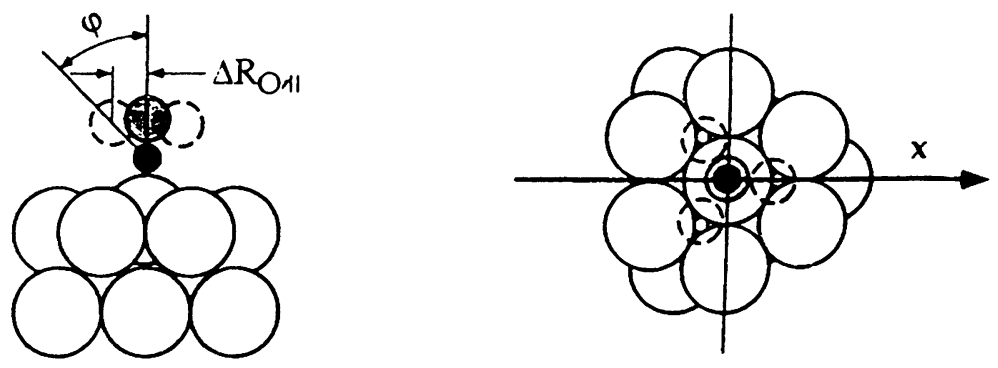

Fig. 2. Illustration of the split positions of the $O$ atom. 6 partially occupied positions and $a$ rotation of the $O$ positions by $30^{\circ}$ have been tried as well leading to the same result. 
We then introduced split positions for both the $\mathrm{O}$ and the $\mathrm{C}$ atom. The split positions for $\mathrm{O}$ are shown in fig. 2. We tried three split positions as well as 6 . Both giving nearly identical results.

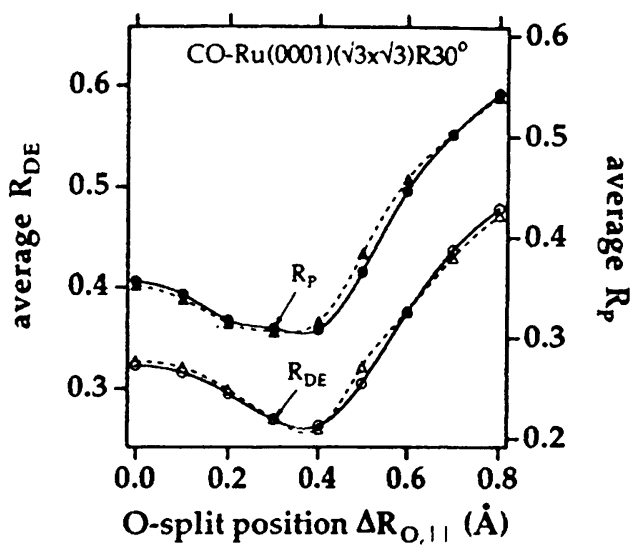

Fig. 3. Average $R$-factor $R_{P}$ and $R_{D E}$ as a function of the $O$ displacement. The dashed line corresponds to the calculation according to equ. 20.

Fig. 3 shows the $\mathrm{R}$-factors as a function of the $\mathrm{O}$ split position. There is a clear minimum visible for about 0.3 to $0.4 \AA$ shift of the oxygen atom. A lateral shift of the $\mathrm{C}$ atom has been tried as well and resulted in a flat minimum at about $0.04 \AA$. In these calculations a Debye temperature of $400 \mathrm{~K}$ has been applied to both $\mathrm{O}$ and $\mathrm{C}$ which takes account of an isotropic contribution and corresponds to a mean square displacement of $0.04 \AA$.

The average tilt amounts to about $15^{\circ}$ if we identify the displacement of $0.3 \AA$ with a mean square displacement. We can compare this result with previous ESDIAD studies on this system by Riedl and Menzel [5,6]. They found a mean square deviation of about $0.1 \AA$ corresponding to about $5^{\circ}$ for the average tilt angle. A considerable larger value found earlier by Madey [7] also with ESDIAD measurements can probably be explained by inappropriate corrections to the ion trajectories due to the static electric field [6]. There remains therefore a factor of 3 between the ESDIAD measurements and the present LEED analysis. This fact should not be overestimated. The average displacement of 0.3 $0.4 \AA$ for $\mathrm{O}$ is certainly above the limit of the applicability of split positions because the exponential in equ. 19 is not small for higher energies and high index beams. The tendency of the split positions is then to overestimate the displacement. The error limits of the 
analysis are therefore large. On the other hand, the interpretation of the ESDIAD measurements is not quite straight forward either and there is an influence of static displacements and defects which has been not been considered in the LEED analysis. Further investigations with temperature dependent measurements and an analysis of the probability density function are in progress.

In summary, we have shown that there is a clear sensitivity of the LEED I/V curves to anisotropic vibrations and the magnitude of amplitude obtained with the simple aproximation with split positions seems to be reasonable though still not quantitatively comparable with other measurements. We can expect that a more realistic calculation of anisotropic thermal vibrations as outlined above offers a convenient and experimentally relative simple way to determine thermal vibrations at surfaces.

\section{Acknowledgement}

We thank Dr. H. Bludau, M. Gierer and T. Hertel for stimulating discussions and Prof. Dr. D. Menzel for providing the LEED data and useful discussions concerning the comparison with ESDIAD measurements.

\section{References}

[1] S. Nagano and S.Y. Tong, Phys. Rev. B 32, 6562 (1985)

[2] Y. Joly, Phys. Rev. :Letters 68, 950 (1992)

[3] H. Over, W. Moritz, and G. Ertl, Phys. Rev. Letters, 70, 315 (1993)

[4] G. Michalk, W. Moritz, H. Pfnür, and D. Menzel, Surf. Sci. 129, 92 (1983)

[5] W. Riedl and D. Menzel, Surf. Sci. 163, 39 (1985)

[6] W. Riedl and D. Menzel, Surf. Sci. 207, 494 (1989)

[7] T.E. Madey, Surf. Sci. 79, 575 (1979)

[8] J.B. Pendry, Low energy electron diffraction, Academic Press 1974

[9] C.B. Duke and G.E. Laramore, Phys. Rev. B 2, 4765 (1970)

[10] B.W. Holland, Surf. Sci. 28, 258 (1971)

[11] M. Ulehla and H.L.Davis, J. Vac. Sci. Technol. 15, 642 (1978)

[12] J.B. Pendry and K. Heinz, Surf. Sci. 230, 137 (1990) 